\title{
Ideal glass-glass transitions and logarithmic decay of correlations in a simple system.
}

\author{
L. Fabbian, W. Götze * , F. Sciortino, P. Tartaglia, F. Thiery \\ Dipartimento di Fisica, Università di Roma La Sapienza \\ and Istituto Nazionale di Fisica della Materia, Unità di Roma La Sapienza \\ Piazzale Aldo Moro 2, I-00185 Roma,Italy
}

(August 19, 2019)

\begin{abstract}
We calculate the ideal-glass-transition line for adhesive hard spheres in the temperature-volumefraction plane within the framework of the mode-coupling theory. We find two intersecting lines, controlled by the hard-core and the adhesive part of the potential respectively, giving rise to two different mechanisms for structural arrest. In the glass region we identify the presence of a glassglass-transition line ending in a cusp bifurcation which causes, even in the close by liquid region, a logarithmic decay of correlations.
\end{abstract}

PACS numbers: 64.70.Pf, 82.70.Dd

The crossover from a liquid to an amorphous solid, observed near the calorimetric glass transition temperature $T_{g}$, exhibits as a precursor phenomenon an anomalous dynamics, called glassy dynamics. Its evolution is connected with a critical temperature $T_{c}$ above $T_{g}$. It has been studied extensively in the recent literature of the glass-transition problem, both experimentally [1] 5 , numerically [6,7] and theoretically [8,9]. Experiments around $T_{c}$ have been interpreted in the frame of the mode-coupling theory (MCT) for structural relaxation. MCT deals primarily with closed equations of motion for the normalized density-fluctuation-correlation functions $\Phi_{q}(t)$ for wavevector moduli $q$. The equilibrium structure enters as input in these equations via the static structure factor $S_{q}$. The theory explains $T_{c}$ as a glass-transition singularity resulting as a bifurcation phenomenon for the self-trapping problem of density fluctuations. Below $T_{c}$ the interaction of density fluctuations leads to arrest in a disordered array, characterized by a Debye-Waller factor $f_{q}>0$. Near the transition, the MCT equations can be solved by asymptotic expansions. Beyond the initial transient dynamics, correlation functions are predicted to decay with a time fractal toward a plateau value $f_{q}^{c}$, the critical Debye-Waller factor. Above $T_{c}$ the correlations decay from $f_{q}^{c}$ to zero, and this is the MCT interpretation of the $\alpha$-process of the classical literature of glassy dynamics [10]. The initial part of this decay is another time fractal, called von Schweidler's law. The values of the fractal exponents are controlled by the so-called exponent parameter $\lambda \leq 1$, which depends solely on $S_{q}$. For details and citations of the original literature the reader is referred to the review in Ref. [9]. A number of tests of MCT results against data, among them the ones in Refs. [1 ] 0, 11,12], demonstrates that this theory treats reasonably the evolution of structural relaxation in some systems.

The MCT bifurcations are caused by a nondegenerate eigenvalue of a certain stability matrix to approach unity from below [13]. Therefore the bifurcation scenario for $f_{q}$ is that known for the zeroes of a polynomial as induced by changes of the polynomial's coefficients [14]. The generic case for a change due to a single control parameter is a fold bifurcation [14], i.e. the coalescence of two real zeroes to a degenerate one. In all the microscopic models for liquid-glass transitions studied so far, only the fold bifurcation singularity had been identified. The next more complicated scenario is the cusp bifurcation, equivalent to the coalescence of three real zeroes. Generically, this case can be obtained only if two control parameters are varied 114. The MCT-bifurcation dynamics for this case has been worked out in a leading order asymptotic expansion [15]. It is drastically different from the one for a fold bifurcation in the sense that relaxation stretching is much more pronounced. In particular there appear logarithmic decay laws, i.e. $1 / f$-noise spectra. Cusp bifurcations in MCT are endpoints of transition lines characterized by $\lambda=1$, and typically they are located near intersections of bifurcation lines [13]. Sjögren [16] identified some sets of polymer dielectric-loss spectra which could not be fitted by the fold-scenario but were compatible with a cusp bifurcation. However, in the data used in Ref. 16 the temperature was varied as the only control parameter, and therefore a complete test of the cusp-bifurcation scenario was not possible.

In this paper we want to demonstrate that a cusp bifurcation near the intersection of two fold-bifurcation lines is possible in a simple but realistic model, if the two conventional control parameters temperature $T$ and density $n$ are varied. With this aim we study an extension of the hard sphere system (HSS), the sticky hard spheres sys-

* Permanent address: Physik-Department, Technische Universität München, 85747 Garching, Germany 
tem (SHSS) introduced by Baxter [17 and used extensively, in particular in colloid physics 18,19. The HSS is the archetype of a simple system and its only control parameter is the packing fraction $\phi=\pi n \sigma^{3} / 6$, with $\sigma$ denoting the hard sphere diameter. It has been studied by dynamic light scattering in its realization as a certain colloidal suspension. The system exhibits an ideal glass transition at some critical packing fraction $\phi_{c}$,4. This is a sharp transition of the fully equilibrated sample from an ergodic liquid to a nonergodic amorphous solid. The glassy dynamics of the colloid exhibits the same scaling laws and $f_{q}$ anomaly as found for the more conventional systems [1 3 , 6, [7, 11, 12]. The measured correlators can be explained quantitatively by the first-principle calculation produced by MCT [5]. The SHSS potential is the limiting form of a square well, infinitely narrow and infinitely deep [20]. It is defined by the interparticle potential $\beta V(r)=\infty$ for $r \leq \sigma, \ln (12 \tau \Delta /(\sigma+\Delta))$ for $\sigma<r \leq \sigma+\Delta$, and 0 for $r>\sigma+\Delta$, in the limit $\Delta \rightarrow 0$. Here $\beta=\left(k_{B} T\right)^{-1}, k_{B}$ is Boltzmann's constant, and $\tau$ is the adhesiveness parameter which plays the role of a dimensionless temperature. For $\tau \rightarrow \infty$ one recovers the HSS. The Ornstein-Zernike equation $S_{q}=1 /\left(1-n c_{q}\right)$ with the Percus-Yevick closure has been solved analytically by Baxter and we use his expressions for $S_{q}$, as a function of $\tau$ and $\phi$ [17 as input of this work. The crucial point is that the interaction exhibits two features, which favour arrest of density fluctuations for two quite different reasons. One is a strong short-ranged repulsion. It is the mechanism leading to the cage effect for liquid dynamics and thereby to arrest driven by density fluctuations near the structure factor peak position. The other one is a short-ranged attraction. It leads via the usual mean-field mechanism to a softening of the density fluctuations, in particular of those for small wave vectors. It is the mechanism which leads to a liquid-gas transition at low values of $\tau$.

The MCT equations of motions are [9]

$\ddot{\Phi}_{q}(t)+\nu_{q} \dot{\Phi}_{q}(t)+\Omega_{q}^{2} \Phi_{q}(t)+\Omega_{q}^{2} \int_{0}^{t} d s m_{q}(t-s) \dot{\Phi}_{q}(s)=0$.

Here $\Omega_{q}=\sqrt{q v / S_{q}}$, with $v$ denoting the thermal velocity, is an effective phonon-dispersion law and $\nu_{q}=\nu_{1} q^{2}$ denotes a damping constant (we use $\nu_{1}=1$ ). The kernel $m_{q}$ is given as $m_{q}(t)=\mathcal{F}_{q}\left[\Phi_{k}(t)\right]$, where the mode-coupling functional $\mathcal{F}_{q}$ is determined by the structure factor:

$$
\begin{gathered}
\mathcal{F}_{q}\left[f_{k}\right]=\frac{1}{2} \int \frac{d^{3} k}{(2 \pi)^{3}} V_{\vec{q}, \vec{k}} f_{k} f_{|\vec{q}-\vec{k}|}, \\
V_{\vec{q}, \vec{k}} \equiv S_{q} S_{k} S_{|\vec{q}-\vec{k}|} \frac{n}{q^{4}}\left[\vec{q} \cdot \vec{k} c_{k}+\vec{q} \cdot(\vec{q}-\vec{k}) c_{|\vec{q}-\vec{k}|}\right]^{2} .
\end{gathered}
$$

We numerically solved Eq. (11) on a grid of 400 equally spaced $q$ values extending up to $q \sigma=72$ [21]. $f_{q}$ is obtained by an iterative solution of the bifurcation equation

$$
f_{q} /\left(1-f_{q}\right)=\mathcal{F}_{q}\left[f_{k}\right]
$$

For $\tau \rightarrow \infty$ we recover the known HSS result, for which the ideal glass transition at $\phi_{c}^{H S S} \sim 0.516$ [ 8$]$ is lead by the increase of $-c_{q}$ and $S_{q}$ with $\phi$. For $\phi \geq \phi_{c}^{H S S}$, the particles are trapped in cages formed by their neighbors. On decreasing $\tau$ attractive forces become relevant; nearest neighbor pairs are closer and therefore holes in the cage are produced, destabilizing the glass. A decrease of $\tau$ has to be compensated by an increase of $\phi$ and thus the $\tau_{c}$ versus $\phi_{c}$ high-temperature-transition line has to bend away from the $\phi=\phi_{c}^{H S S}$ value. This effect is brought out by the MCT solutions as shown by the dashed lines $B_{1}$ in Fig. 1. The destabilization of the glass on decreasing $\tau$ is related, within $\mathrm{MCT}$, to the increase of pair correlations which weaken the screened potential $-c_{q}$ and decrease the value of $S_{q_{\max }}$, as shown in Fig. 2-top. Moving along $B_{1}$ in Fig. 1 by decreasing $\tau$ implies an increase of the attraction and thus of the particle localization compared to the HSS case. Consequently, $f_{q}^{c}$ increases and spreads out further in the $q$-domain as demonstrated in Fig. 2 top.

The increase of $c_{q}$ and $S_{q}$ at small $q$ becomes increasingly relevant on decreasing $\tau$ producing a different mechanism for structural arrest. This increase of the compressibility is a precursor of the liquid-gas critical point. The enhanced amplitudes for density fluctuations increase their anharmonic interactions, and indeed the solution of the MCT equations predict their spontaneous arrest on a low-temperature-transition line $B_{2}$ in the $\tau$ vs $\phi$ plane, shown in Fig. 11. Increase of $\phi$ suppresses small $q$ fluctuations. But it also increases $-c_{q}$ and the first peak of $S_{q}$, as shown by a comparison of results shown in Fig. 2-middle. Both effects stabilize the glass, since the mode-coupling constants, Eq. (3) increase. This explains the upward bending of the full line in Fig. 1. Since arrest for small $q$ is favored on the $B_{2}$ branch, $f_{q}^{c}$ is much larger there than for the HSS. The decrease of the compressibility upon increasing $\phi$ make spontaneous arrest less easy and therefore $f_{q}^{c}$ decreases upon compressing. Both features are demonstrated in Fig.2-middle.

The explained interplay of attraction, and repulsion places the meeting point $C^{*}$ of the two branches $B_{1}$ and $B_{2}$ to a packing fraction $\phi^{*}=0.5565$, higher than $\phi_{c}^{H S S}$, and to $\tau^{*}=1.320$. Thus there is a reentry phenomenon if $\tau$ is lowered, e.g. for a packing fraction between 0.52 and 0.55 . At some $\tau$ above 1.32 the glass melts since the increase of attraction-induced-pair-correlations destabilizes the cages for particle arrest. At some lower $\tau$ the liquid freezes again into a glass, because of the compressibility increase. At $C^{*}$, the $f_{q}$ calculated on the two different branches, $f_{q}^{(1)}$ and $f_{q}^{(2)}$, are different, while the corresponding $S_{q}$ are indistinguishable. Thus two types 
of glasses, differing in their $f_{q}$ and in their dynamical properties, can be obtained close to $C^{*}$. In the region where both $f_{q}^{(1)}$ and $f_{q}^{(2)}$ are solutions of the Eq. (4), the one with the larger $f_{q}$ is the relevant solution. This exemplifies the maximum theorem of MCT: if there is a solution of Eq. (ब), say $\tilde{f}_{q}$, the long time limit $f_{q}$ at the same equilibrium state obeys $f_{q} \geq \tilde{f}_{q}[13]$. For our case it means that the continuation of $f_{q}^{c(2)}$ takes over the role of $f_{q}$, i.e. $B_{1}$ stops at $C^{*}$. Therefore the two branches of the transition line do not join smoothly at $C^{*}$. The $B_{2}$ branch continues, as shown by the inset of Fig. 1 i until it reaches a cusp bifurcation as an endpoint of the transition line. Indeed, we evaluate the exponent parameter $\lambda$ along the $B_{1}$ and $B_{2}$ lines and find, as shown in the inset of Fig. 2-bottom, that along the $B_{2}$ line $\lambda$ keeps on increasing till it reaches unity for $\tau=1.37$ and $\phi=0.55807$. The line between $C^{*}$ and the cusp point is a line of glass-to-glass transitions. For the given equilibrium structure for $(\tau, \phi)$ on this line, there are two possibilities for structural arrest. The one on high $\tau$ side is driven by the excluded volume mechanism and the other one by the attraction-induced compressibility increase. The latter mechanism leads to a larger $f_{q}$ than the former. The endpoint is characterized by their difference approaching zero, as shown in Fig. 2-bottom.

In Fig. 3 the glassy dynamics outside the transient is exhibited for states in the reentry region. The dynamics is, up to a regular time scale, independent of the transient. In particular it is the same for a colloid, as for a conventional liquid for which Eq. (1) is formulated [9]. The uppermost curve corresponds to a point in the glass region and demonstrates arrest near the $B_{1}$ branch, the others exhibit stretched liquid relaxation to zero. Interestingly enough, the exhibited anomalous dynamics is dominated by the cusp singularity, not by the closer fold. Indeed even though a huge dynamical window is considered, the known fold bifurcation pattern cannot be recognized. There is no power-law decay towards the $f_{q}^{c(1)}$ nor is there an $\alpha$-process obeying the superposition principle. We checked that the mentioned asymptotic fold-bifurcation features appear only after tuning $\phi$ much closer to the transition line, which implies the extension of the dynamical window to even larger sizes. Instead, the cusp dynamics appears in the liquid region. The stretched relaxation in the three-decade window $0 \leq \log _{10}\left(t / t_{0}\right) \leq 3$ shows the approach towards the critical decay of the cusp [15]. Then the correlators follow closely a logarithmic law in time. For $\phi=0.555$ it extends over 5 decades. Upon decreasing $\phi$ its range of validity shrinks but even at $\phi=0.540$ it extends over two decades. This logarithmic decay is the known cusp substitute for the start of a fold $\alpha$-process. Let us emphasize that the above scenario does not require a fine tuning of $\tau$. For $\tau=1.32$ the pattern is similar to the one shown for $\tau=1.40$.
In summary, we have shown that the SHSS presents a peculiar structural arrest dynamics at high packing fractions due to the competing mechanism of hard core and attractive interactions. Two differently sloped idealglass transition lines appear in the phase diagram. In the region where these two lines meet the slow dynamics changes from power-law to a logarithmic-law due to the influence of a nearby cusp singularity. In most tests of MCT the data had been compared with the universal results obtained for the asymptotic dynamics near a fold transition. But our results show that even a simple system can exhibit glassy dynamics, which does not exhibit asymptotic laws within accessible dynamical windows. It seems relevant to test, by spectroscopy for colloidal systems and by molecular-dynamics simulations, whether specific systems exhibit the shown complex dynamics and, in case, whether it is handled properly by the present MCT calculation.

[1] H. Z. Cummins, G. Li, W. Du, Y.H. Hwang, and G. Q. Sheu, Prog. Theor. Phys. 126, 21 (1997).

[2] A. Tölle, H. Schober, J. Wuttke, and F. Fujara, Phys. Rev. E 56, 809 (1997).

[3] W. Petry, E. Bartsch, F. Fujara, M. Kiebel, H. Sillescu, and B. Farago, Z. Phys. B83, 175 (1991).

[4] W. van Megen and P. N. Pusey, Phys. Rev. A 43, 5429 (1991).

[5] W. van Megen and S. M. Underwood, Phys. Rev. E 49, 4206 (1994).

[6] W. Kob and H. C. Andersen, Phys. Rev. E 51, 4626 (1995).

[7] F. Sciortino, P. Gallo, P. Tartaglia, S.-H. Chen, Phys. Rev. E 546331 (1996); F. Sciortino, L. Fabbian, S.-H. Chen and P. Tartaglia, Phys. Rev. E 56, 5397 (1997).

[8] U. Bengtzelius, W. Götze, and A. Sjölander, J. Phys. C 17, 5915 (1984).

[9] W. Götze and L. Sjögren, Rep. Prog. Phys. 55, 241 (1992).

[10] C. A. Angell, in Complex Behavior of Glassy Systems, M. Rubí and C. Pérez-Vicente eds, (Springer, Berlin, 1997) p. 1.

[11] M. Nauroth and W. Kob, Phys. Rev. E 55, 657 (1997).

[12] L. Fabbian, F.Sciortino, F. Thiery, and P. Tartaglia, Phys. Rev. E 57, 1485 (1998).

[13] W. Götze, Liquids, Freezing and Glass Transition, ed. by J.-P. Hansen, D. Levesque, and J. Zinn-Justin (NorthHolland, Amsterdam, 1991) p. 287.

[14] V. I. Arnold, Catastrophe Theory, 3rd ed. (Springer Verlag, Berlin, 1992).

[15] W. Götze and L. Sjögren, J. Phys.: Condensed Matter 1, 4203 (1989).

[16] L. Sjögren, J. Phys.: Condensed Matter 3, 5023 (1991).

[17] R. J. Baxter, J. Chem. Phys. 49, 2770 (1968).

[18] S. H. Chen, J. Rouch, F. Sciortino and P. Tartaglia, J. 
Phys.: Condens. Matter 6, 10855 (1994).

[19] H. Verduin and J. K. G. Dhont, J. Coll. Int. Sc. 172, 425 (1995).

[20] Using a perturbative solution of the square well potential of depth $V_{0}$ and width $\Delta$ in the parameter $\epsilon=\Delta /(\sigma+\Delta), \tau$ can be related to temperature as $\tau^{-1}=12 \epsilon \exp \left(-\beta V_{0}\right)$. See S. V. G. Menon, C. Manohar, K. Srinivasa Rao, J. Chem. Phys. 95, 9186 (1991).

[21] F. Thiery, Thesis, University of Rome La Sapienza (unpublished). 


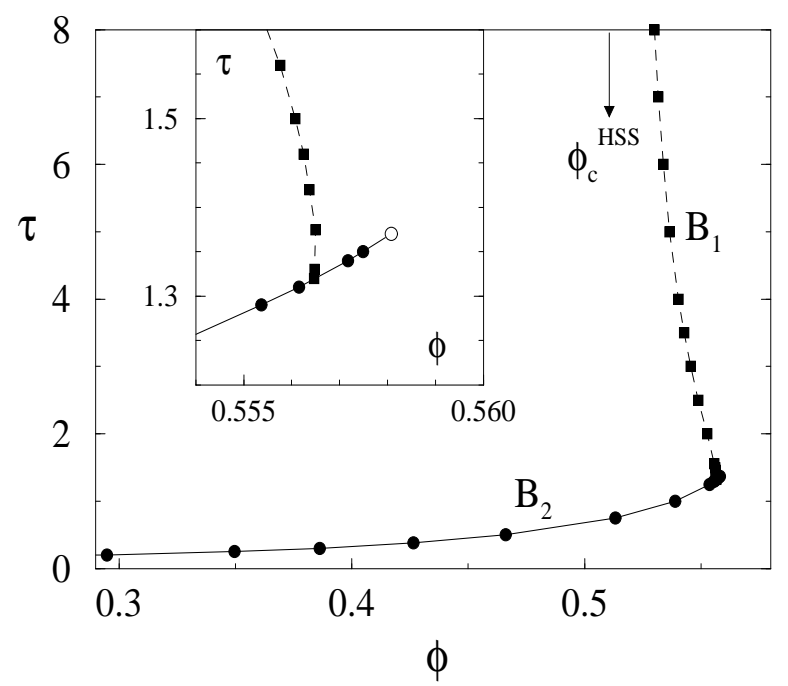

FIG. 1. Ideal glass-transition lines for the Baxter model (squares and dots). $B_{1}$ (dashed) is the high temperature branch of the glass-transition line and $B_{2}$ (full) is the low temperature one. The arrow indicates the critical packing fraction of the hard sphere system. The open circle denotes the cusp-point. 


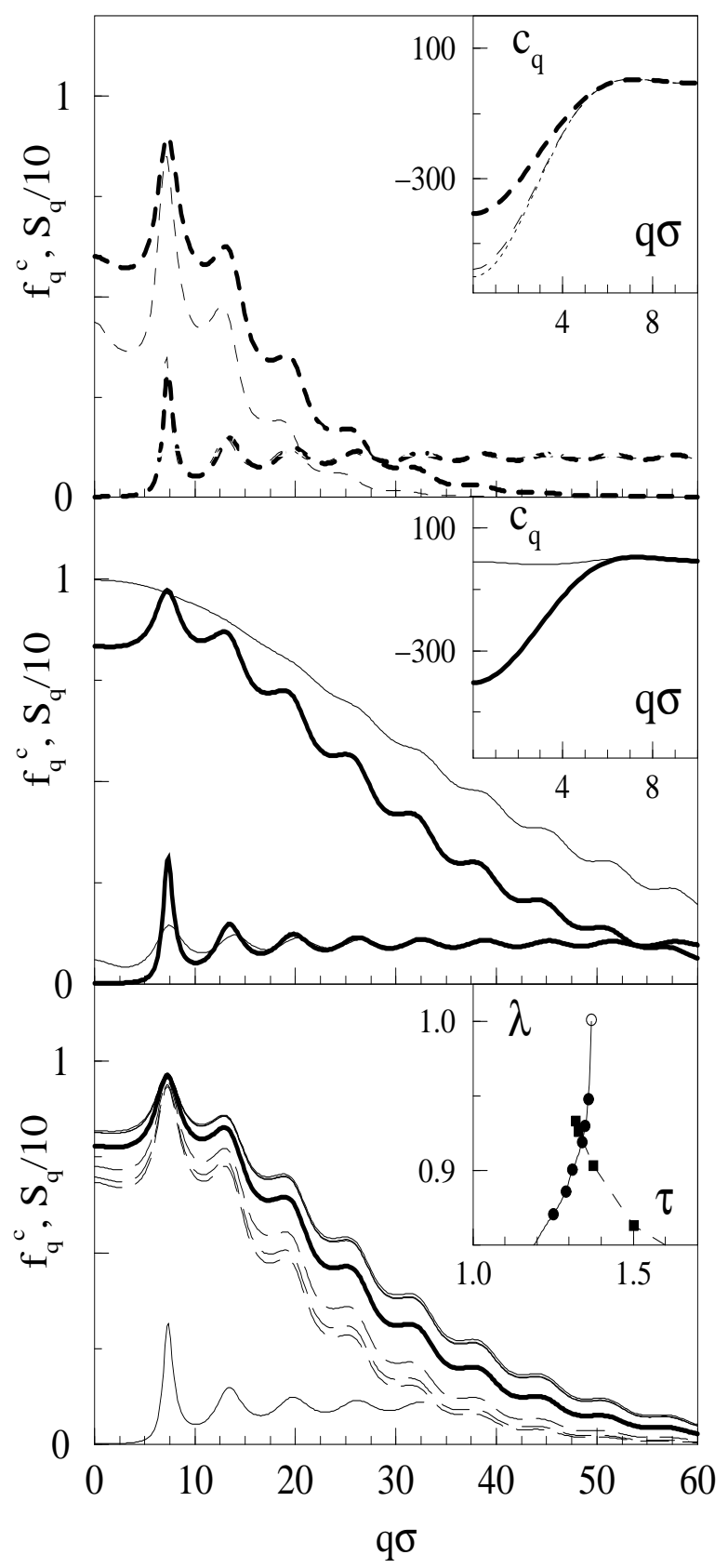

FIG. 2. Structure factors $S_{q} / 10$, direct correlation functions $c_{q}$ and critical Debye-Waller factors $f_{q}^{c}$ along $B_{1}$ (top), $B_{2}$ (middle) and along the glass-glass transition line (bottom). Top: $\tau_{c}=8, \phi_{c}=0.530$ (light dashed lines) and $\tau_{c}=1.32$, $\phi_{c}=0.522$ (heavy dashed lines). The dotted line in the inset is $c_{q}$ at $\phi_{c}^{H S S}=0.516$ for $\tau=\infty$. Middle: $\tau_{c}=0.2$, $\phi_{c}=0.295$ (light full lines) and $\tau_{c}=1.31, \phi_{c}=0.5561$ (heavy full lines). Bottom: The full (dashed) lines refer from top to bottom (bottom to top) to $\tau=1.34,1.35,1.36$ respectively, on the $B_{2}\left(B_{1}\right)$ side of the glass-glass transition line. The heavy full line is $f_{q}$ for the cusp point $\tau=1.37, \phi=0.55807$. The lower $S_{q}$ refers to the same states. The inset shows the exponent parameter $\lambda$ along $B_{1}$ (dashed) and $B_{2}$ (full).

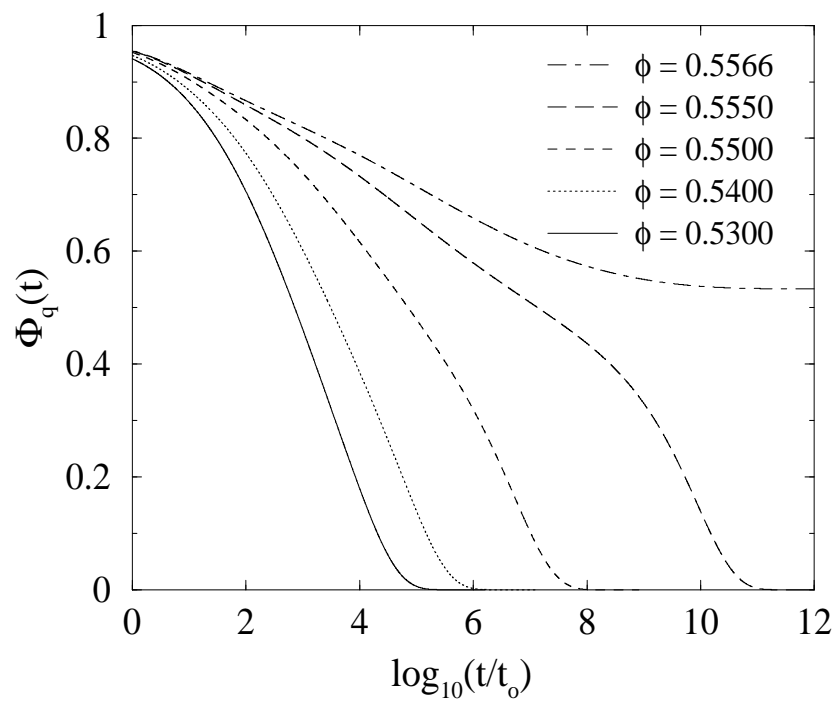

FIG. 3. Density correlator for $q \sigma=14.4$ at $\tau=1.40$ and various $\phi$ as a function of $\log _{10}\left(t / t_{0}\right)$ with $t_{0}=\sigma / v$. 